\title{
Endocytotic Process
}

National Cancer Institute

\section{Source}

National Cancer Institute. Endocytotic Process. NCI Thesaurus. Code C16544.

Cellular uptake of extracellular materials within membrane-limited vacuoles or microvesicles. Endosomes play a central role in endocytosis. 